\title{
Metastatic Adrenal Gland Pheochromocytoma
}

National Cancer Institute

\section{Source}

National Cancer Institute. Metastatic Adrenal Gland Pheochromocytoma. NCl

Thesaurus. Code C8046.

A pheochromocytoma that has metastasized to other anatomic sites. 